\title{
Queueing Systems with Random Volume Customers and their Performance Characteristics
}

\author{
Oleg Tikhonenko \\ Institute of Computer Science \\ Cardinal Stefan Wyszyński University in Warsaw, \\ Warsaw, Poland
}

o.tikhonenko@uksw.edu.pl

\author{
Marcin Ziółkowski \\ Institute of Information Technology \\ Warsaw University of Life Sciences - SGGW, \\ Warsaw, Poland
}

marcin_ziolkowski@sggw.pl

\begin{abstract}
In the paper, we consider non-classical queueing systems with non-homogeneous customers. The non-homogeneity we treat in the following sense: in systems under consideration, we characterize each customer by random capacity (volume) that can have an influence on his service time. We analyze a stochastic process having the sense of the total volume of all customers present in the system at given time instant. Such analysis for different queueing systems with unlimited or limited total volume can be used in designing of nodes of computer and communication networks while determining their buffer space capacity. We discuss basic problems of the theory of these systems and their performance characteristics. We also present some examples and results for systems with random volume customers.

Keywords: customer's volume, buffer space capacity, total volume, loss probability, volume unit loss probability, Laplace-Stieltjes transform
\end{abstract}

\section{Introduction}

Classical queueing theory was created by Danish engineer and scientist A.K. Erlang in the early years of $20^{\text {th }}$ century. Its first mathematical models were used in designing of telephone networks. Now, they are known as $M / M / n / 0$ or Erlang systems. Nowadays, queueing theory contains a set of developed mathematical tools that are widely used as mathematical models of many real objects, such as markets, communication and computer networks, airport traffic, Internet etc. (see e.g. [1], [2], [3], [4], [5]).

Classical queueing models are often used for determination of communication networks' nodes characteristics [6], [7], [8], [9]. One of the most important of them is loss probability or probability of message's losing that depends on messages' arrival process characteristics, buffer space capacity of the node and distributions of message's volume and its service time. 
Let $\eta(t)$ be the number of customers present in a system under consideration at time instant $t$. It is known that, in classical queueing theory, the determination of almost all performance characteristics comes to analysis of a stochastic process $\eta(t)$. But such analysis becomes insufficient, if we want e.g. to determine buffer space capacity of a communication network's node that guarantees small losses of information transmitted in the network. The causes of this fact are the following: 1) different messages (customers) in a network consist, as a rule, of different numbers of bytes, in other words, they have different volumes; 2) service time of a customer can be dependent on his volume; 3 ) the total (summarized) volume of customers present in the system is limited by some buffer capacity. The factors $1-3$ are not be able taken into consideration in classical queueing theory.

To overcome appeared difficulties, we have to introduce the following assumptions: 1) each customer in the system is characterized by random volume $\zeta$, where $\zeta$ is a non-negative random variable (RV);2) service time $\xi$ of a customer can depend on his volume, generally, this dependence can be defined by a joint distribution function (DF)

$$
F(x, t)=\mathbf{P}\{\zeta<x, \xi<t\}
$$

Let $\sigma(t)$ be the total volume, i.e. the sum of volumes of all customers present in the system at time instant $t$. The process $\sigma(t)$ is a generalization of $\eta(t)$ one. Indeed, their values are the same, if $\zeta \equiv 1$. It is clear that changing epochs of both these processes are the same, because their values change at epochs of customers arriving to the system and customers service termination. But it is clear that an analysis of the process $\sigma(t)$ is more complicated, because, at an epoch of service termination, each customer takes away from the buffer the same volume that he brings to it at an arrival epoch. For the process $\eta(t)$, these values are equal 1 and -1 , respectively. We assume that volumes of different customers are independent and do not depend on the customers' arriving epochs.

Note that, from relation (1), we obtain the following formulae for DFs $L(x)$ and $B(t)$ of $\operatorname{RVs} \zeta$ and $\xi$, respectively:

$$
L(x)=\mathbf{P}\{\zeta<x\}=\lim _{t \rightarrow \infty} F(x, t)=F(x, \infty), B(t)=\mathbf{P}\{\xi<t\}=\lim _{x \rightarrow \infty} F(x, t)=F(\infty, t) .
$$

In queueing models of real systems, the total volume is usually limited by constant value $V>0$ that is called buffer space capacity of the system. Below, we assume that inequality $0 \leq \sigma(t) \leq V$ holds (the case of $V=\infty$ is also possible for some models). We also assume that buffer space is occupied by a customer at an epoch he arrives and is released entirely at an epoch he completes service. If the value $V$ is finite, it leads to additional losses of customers. A customer of volume $x$, which arrives at epoch $\tau$ when there are idle servers or waiting positions in the queue, will be admitted to the system, if $\sigma(\tau-0)+x \leq V$, where $\sigma(\tau-0)$ is the left-sided limit of the process $\sigma(t)$ at the point of $\tau$. In other words, if $\tau$ is an arriving epoch, then $\sigma(\tau-0)$ is the number of customers present in the system just before $\tau$. Otherwise (if $\sigma(\tau-0)+x>V$ ), the customer will be lost. In the first case, we have

$$
\eta(\tau)=\eta(\tau-0)+1, \sigma(\tau)=\sigma(\tau-0)+x,
$$


where $\eta(\tau-0)$ is the left-sided limit of the process $\eta(t)$ at the point of $\tau$. In the second case, we obtain:

$$
\eta(\tau)=\eta(\tau-0), \sigma(\tau)=\sigma(\tau-0) .
$$

If $t$ is an epoch of service termination of a customer having volume $x$, we obtain

$$
\eta(t)=\eta(t-0)-1, \sigma(t)=\sigma(t-0)-x .
$$

\section{Classification of Models}

Consider firstly a classical queueing system of $G I / G / n / m(1 \leq n \leq \infty, 0 \leq m \leq \infty)$ type in Kendall's notation (see e.g. [2]). This system with identical servers $S_{1}, \ldots, S_{n}$ is shown schematically in Fig. 1.
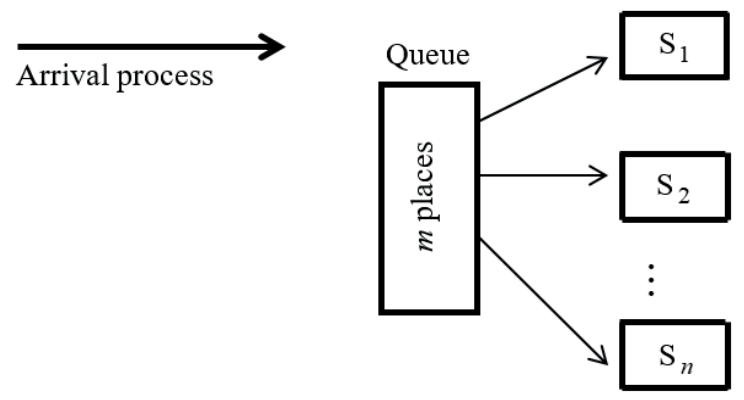

Fig. 1. Classical queueing system $G I / G / n / m$

Denote by $G I / G / n /(m, V)$ a similar system with random volume customers and limited by value $V(V \leq \infty)$ total volume. It is shown in Fig. 2 .
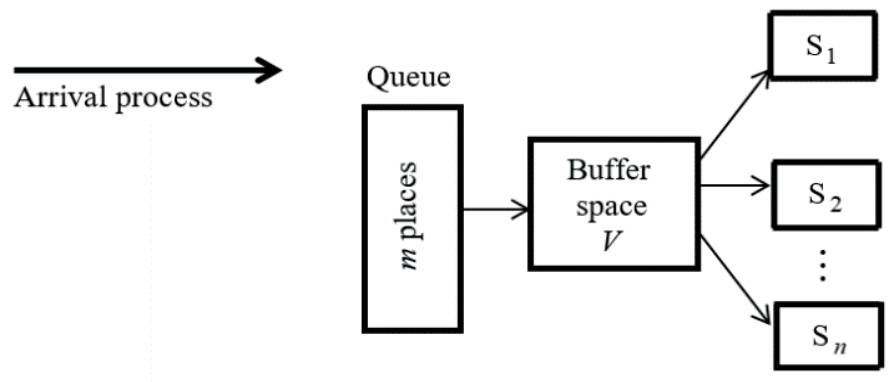

Fig. 2. Queueing system $G I / G / n /(m, V)$ with random volume customers

It is clear that the system shown in Fig. 2 generally differs from classical one, because its loss characteristics at some arrival epoch depend on the volume of arriving customer and the total volume of other customers present in the system at this time. Indeed, compare mechanisms of customers' losing in both systems under assumption that there are no other limitations except those shown in Fig. 1 and Fig. 2.

In both systems, a customer is lost, if, at the epoch $\tau$ of his arrival, there are $n+m$ other customers in each of them $(\eta(\tau-0)=n+m)$. In the system $G I / G / n /(m, V)$, 
a customer having volume $x$ is lost not only if $\eta(\tau-0)=n+m$, but also if $\sigma(\tau-0)+$ $x>V$. It means that an admission or losing of a customer partly or completely depends on his volume and total volume of other customers present in the system.

Classification of models of systems with random volume customers is determined by the type of RVs $\zeta$ and $\xi$ dependence and the value $V$. Then, we can distinguish four following classes of these models: 1) models with $V=\infty$ and independent RVs $\zeta$ and $\xi(F(x, t)=L(x) B(t)) ; 2)$ models with $V<\infty$ and independent RVs $\zeta$ and $\xi ; 3)$ models with $V=\infty$ and dependent RVs $\zeta$ and $\xi(F(x, t) \neq L(x) B(t)) ; 4)$ models with $V<\infty$ and dependent RVs $\zeta$ and $\xi$.

Note that an analysis of models of the first class can be carried out by tools of classical queueing theory.

Consider e.g. a steady-state queueing system $M / G / 1 / \infty$. Let $L(x)$ be DF of customer's volume $\zeta$, and service time $\xi$ of a customer does not depend on his volume. Denote by $B(t)$ DF of RV $\xi$ and by $\beta(q)=\int_{0}^{\infty} e^{-q t} d B(t)$ Laplace-Stieltjes transform (LST) of this DF. Let $a$ and $\beta_{1}$ be parameters of the arrival process and the mean service time of a customer, respectively, then we have $\rho=a \beta_{1}<1$.

It is clear that, in the system under consideration, a distribution of number of customers $\eta$ present in the system in steady state does not depend on their volumes. Introduce the notation $p_{k}=\mathbf{P}\{\eta=k\}$. Let $P(z)=\sum_{k=0}^{\infty} p_{k} z^{k},|z|<1$, be generating function of number of customers present in the system. Then, the Pollaczek-Khinchine formula takes place (see e.g. [1]):

$$
P(z)=\frac{(1-\rho)(1-z) \beta(a-a z)}{\beta(a-a z)-z} .
$$

Denote by $D(x)$ DF of the total steady-state customers' volume $\sigma$. From the fact that volumes of customers are independent and their service times do not depend on their volumes, we obtain the following relations: $\mathbf{P}\{\sigma<x \mid \eta=k\}=L_{*}^{(k)}(x)$, where $L_{*}^{(k)}(x)$ is $k$-fold Stieltjes convolution of DF $L(x)$, i.e. $L_{*}^{(1)}(x)=L(x), L_{*}^{(k)}(x)=$ $\int_{0}^{x} L_{*}^{(k-1)}(x-u) d L(u)$ for $k=2,3, \ldots$, and $\mathbf{P}\{\sigma=0\}=p_{0}=1-\rho$. Then, for $\operatorname{DF} D(x)$, we obtain

$$
D(x)=p_{0}+\sum_{k=1}^{\infty} p_{k} \mathbf{P}\{\sigma<x \mid \eta=k\}=p_{0}+\sum_{k=1}^{\infty} p_{k} L_{*}^{(k)}(x) .
$$

Passing to LST with respect to $x$ in the last relation, we have:

$$
\begin{gathered}
\delta(s)=\int_{0}^{\infty} e^{-s x} d D(x)=\sum_{k=0}^{\infty} p_{k}(\varphi(s))^{k}=P(\varphi(s))= \\
=\frac{(1-\rho)(1-\varphi(s)) \beta(a-a \varphi(s))}{\beta(a-a \varphi(s))-\varphi(s)},
\end{gathered}
$$

where $\delta(s)$ and $\varphi(s)(s)$ denote LSTs of DFs $D(x)$ and $L(x)$, respectively. I.e. we obtain in this case that a distribution of steady-state total volume $\sigma$ is completely determined 
by distribution of number of customers present in the system $\eta$ and DF $L(x)$ of customer's volume.

The main characteristics of models from classes 2 and 4 are the distribution of number of customers present in the system at arbitrary time instant or in steady state and some loss characteristics (e.g. steady-state loss probability).

For systems belonging to class 2, we can often use tools of classical queueing theory. Indeed, let $q_{k}, k=\overline{0, n+m}$, be steady-state probability that $k$ customers present in the classical system $M / G / n / m, n=1,2, \ldots ; m=0,1, \ldots$. Let $M / G / n /(m, V)$ be a system from class 2 that differs from the classical $M / G / n / m$ system in the following details: 1) each customer is characterized by random volume with DF $L(x) ; 2)$ the total customers' volume is limited by buffer space capacity $V<\infty$. Assume that DFs of service time for both systems are the same. It was proved (see [14], [15]) that steadystate probabilities $p_{k}$ of presence of $k$ customers in the system $M / G / n /(m, V)$ can be determined by the following relation: $p_{k}=C q_{k} L_{*}^{(k)}(V), k=\overline{1, n+m}$, where $C$ can be calculated from the normalization condition: $C=\left[\sum_{k=0}^{n+m} q_{k} L_{*}^{(k)}(V)\right]^{-1}$. Note that this relation takes place also if $m=\infty$, when steady state exists for the classical system.

The main characteristic of models from class 3 is the distribution of total volume at arbitrary time instant or in steady state. Various models of queueing systems with random volume customers can be found in [10], [11], [12], [13].

\section{Simple Examples of Systems with Random Volume Customers}

\subsection{System $M / M / n /(\infty, V)$ with independent customer's volume and service time.}

For this system, we assume that service time does not depend on customer's volume and has an exponential distribution with parameter $\mu>0$, i.e. $B(t)=1-e^{-\mu t}, t>$ 0 . The system was firstly analyzed in [10]. Let $L(x)$ be DF of customer's volume. Denote by $\eta$ and $\sigma$ the number of customers present in the system in steady state and their total volume, respectively. Let $a$ be parameter of the arrival process. For this system were obtained the functions $g_{k}(x)=\mathbf{P}\{\eta=k, \sigma<x\}, k=1,2, \ldots, x \in$ $[0 ; V]$ :

$$
g_{k}(x)= \begin{cases}\frac{(n \rho)^{k}}{k !} p_{0} L_{*}^{(k)}(x), & \text { if } k=\overline{1, n}, \\ \frac{n^{n} \rho^{k}}{n !} p_{0} L_{*}^{(k)}(x), & \text { if } k=n+1, n+2, \ldots,\end{cases}
$$

where $p_{0}=\mathbf{P}\{\eta=0\}, \rho=a /(n \mu)$.

Then, we can easily obtain the following relations for probabilities $p_{k}=\mathbf{P}\{\eta=k\}=g_{k}(V)$ : 


$$
p_{k}=\left\{\begin{array}{l}
\frac{(n \rho)^{k}}{k !} p_{0} L_{*}^{(k)}(V), \text { if } k=\overline{1, n} \\
\frac{n^{n} \rho^{k}}{n !} p_{0} L_{*}^{(k)}(V), \quad \text { if } k=n+1, n+2, \ldots
\end{array}\right.
$$

where $p_{0}$ can be determined from the normalization condition as:

$$
p_{0}=\left[A_{n}(V)+\frac{n^{n}}{n !} B_{n}(V)\right]^{-1},
$$

where

$$
A_{n}(x)=\sum_{k=0}^{n} \frac{(n \rho)^{k}}{k !} L_{*}^{(k)}(x), B_{n}(x)=\sum_{k=n+1}^{\infty} \rho^{k} L_{*}^{(k)}(x) .
$$

Loss probability $P_{\text {loss }}$ for this system is determined by the following relation [10]:

$$
P_{\text {loss }}=1-(n \rho)^{-1} \sum_{k=1}^{n-1} k p_{k}-\rho^{-1}\left(1-\sum_{k=0}^{n-1} p_{k}\right) \text {. }
$$

Now, we can easily obtain $\operatorname{DF} D_{V}(x)$ of the total volume $\sigma$ :

$$
D_{V}(x)=p_{0}+\sum_{k=1}^{\infty} g_{k}(x)=p_{0}\left[A_{n}(x)+\frac{n^{n}}{n !} B_{n}(x)\right], x \in[0 ; V] .
$$

Let us consider a special case of the system when customer's volume is distributed exponentially: $L(x)=1-e^{-f x}, f>0, x>0$. Then, we have

$$
\begin{aligned}
& A_{n}(x)=\sum_{k=0}^{n} \frac{(n \rho)^{k}}{k !}\left[1-e^{-f x} \sum_{i=0}^{k-1} \frac{(f x)^{i}}{i !}\right] \\
& B_{n}(x)=\sum_{k=n+1}^{\infty} \rho^{k}\left[1-e^{-f x} \sum_{i=0}^{k-1} \frac{(f x)^{i}}{i !}\right] .
\end{aligned}
$$

After some transformations, we can present the relation for $B_{n}(x)$ in the form that does not contain infinite sums:

$$
B_{n}(x)=\frac{\rho^{n+1}}{1-\rho}\left[1-e^{-f x} \sum_{i=0}^{n} \frac{(f x)^{i}}{i !}\right]-\frac{\rho}{1-\rho}\left[e^{-(1-\rho) f x}-e^{-f x} \sum_{i=0}^{n} \frac{(\rho f x)^{i}}{i !}\right] .
$$

In the case of one-server system $(n=1)$, we obtain (see [13]):

$$
\begin{gathered}
p_{0}= \begin{cases}\frac{1-\rho}{1-\rho e^{-(1-\rho) f V},} & \text { if } \rho \neq 1, \\
(1+f V)^{-1}, & \text { if } \rho=1 ;\end{cases} \\
P_{\text {loss }}= \begin{cases}\frac{1-\rho}{e^{(1-\rho) f V}-\rho}, & \text { if } \rho \neq 1, \\
(1+f V)^{-1}, & \text { if } \rho=1 ;\end{cases} \\
D_{V}(x)= \begin{cases}\frac{1-\rho e^{-(1-\rho) f x}}{1-\rho e^{-(1-\rho) f V},} & \text { if } x \in[0 ; V], \text { for } \rho \neq 1 ; \\
1, & \text { if } x>V,\end{cases} \\
D_{V}(x)= \begin{cases}\frac{1+f x}{1+f V}, & \text { if } x \in[0 ; V], \text { for } \rho=1 . \\
1, & \text { if } x>V\end{cases}
\end{gathered}
$$




\subsection{System $M / G / 1 / \infty$ with unlimited buffer space and service time generally dependent on customer's volume}

Denote by $F(x, t)$ a joint DF of customer's volume $\zeta$ and his service time $\xi$. Let $\alpha(s, q)=\int_{0}^{\infty} \int_{0}^{\infty} e^{-s x-q t} d F(x, t)$ be double LST of DF $F(x, t)$. Then, $\varphi(s)=$ $\alpha(s, 0)=\int_{0}^{\infty} e^{-s x} d L(x)$ and $\beta(q)=\alpha(0, q)=\int_{0}^{\infty} e^{-q t} d B(t)$ are LSTs of DFs $L(x)$ and $B(t)$, respectively. Let $\beta_{1}=\mathbf{E} \xi$ be the mean value of service time. Assume that $\rho=a \beta_{1}<1$, where a is parameter of arrival process. Then, for the system under consideration, finite steady-state characteristics exist. E.g. we have $\sigma(t) \Rightarrow \sigma$ and $\eta(t) \Rightarrow \eta$ in the sense of a weak convergence, where $\sigma$ and $\eta$ are the steady-state total customers' volume and number of customers in the system, respectively. Let $D(x)$ be DF of $\operatorname{RV} \sigma$. Denote by $\delta(s)$ its LST. Then, we obtain [10], [11], [12], [13]:

$$
\delta(s)=\int_{0}^{\infty} e^{-s x} d D(x)=\frac{(1-\rho)[\beta(a-a \varphi(s))-\alpha(s, a-a \varphi(s))]}{\beta(a-a \varphi(s))-\varphi(s)} .
$$

Note that, in the case of independent RVs $\zeta$ and $\xi(F(x, t)=L(x) B(t), \alpha(s, q)=$ $=\varphi(s) \beta(q)$ ), relation (2) follows from (7).

Using relation (7), we can calculate moments of $\mathrm{RV} \sigma$ (if they exist). For the first and second ones, we have [12], [13]:

$$
\begin{gathered}
\delta_{1}=E \sigma=-\delta^{\prime}(0)=a \alpha_{11}+\frac{a^{2} \beta_{2} \varphi_{1}}{2(1-\rho)^{\prime}} \\
\delta_{2}=\mathbf{E} \sigma^{2}=\delta^{\prime \prime}(0)= \\
=a\left(\alpha_{21}+a \varphi_{1} \alpha_{12}\right)+\frac{a^{3} \beta_{2} \varphi_{1} \alpha_{11}}{1-\rho}+\frac{a^{2} \beta_{2} \varphi_{2}}{2(1-\rho)}+\frac{a^{3} \beta_{3} \varphi_{1}^{2}}{3(1-\rho)}+\frac{a^{4} \beta_{2}^{2} \varphi_{1}^{2}}{2(1-\rho)^{2}},
\end{gathered}
$$

where $\varphi_{i}, \beta_{i}$ are the $i$ th moments of $\operatorname{RVs} \zeta$ and $\xi$,respectively, $i=1,2, \ldots, \alpha_{i j}$ is the mixed $(i+j)$ th moment of the random vector $(\zeta, \xi), i, j=1,2, \ldots$

Assume e.g. that customer's volume $\zeta$ has an exponential distribution with parameter $f$, and service time of the customer is proportional to his volume $(\xi=c \zeta, c>0)$. In this case, we can determine an explicit form of DF $D(x)[13]$ :

$$
\begin{gathered}
D(x)=1+\frac{\rho^{2} e^{-(1-\rho) f x}}{1-2 \rho}-\frac{\rho(1-\rho)}{\sqrt{\rho(4+\rho)}}\left(\frac{1-b_{1}}{1-b_{1}-\rho} e^{-b_{1} f x}-\frac{1-b_{2}}{1-b_{2}-\rho} e^{-b_{2} f x}\right), \text { if } \rho \neq 1 / 2,(8) \\
D(x)=1+\frac{1}{9} e^{-2 f x}-\frac{1}{3}\left(\frac{11}{6}+\frac{f x}{4}\right) e^{-f x / 2}, \text { if } \rho=1 / 2
\end{gathered}
$$

where $\rho=a c / f$,

$$
b_{1}=\frac{2+\rho-\sqrt{\rho(4+\rho)}}{2}, b_{2}=\frac{2+\rho+\sqrt{\rho(4+\rho)}}{2} .
$$

Note that, in the case of independent RVs $\zeta$ and $\xi$ when they have exponential distributions with DFs $L(x)=1-e^{-f x}$ and $B(t)=1-e^{-\mu t}$, respectively, we have:

$$
D(x)=1-\rho e^{-(1-\rho) f x},
$$

where $\rho=a / \mu$. 
Note that, in the case of exponential distribution of RV $\zeta$ and the same dependence between $\zeta$ and $\xi(\xi=c \zeta)$, we can also determine an explicit form of $D(x)$ for the system $M / M / n / m$ with unlimited buffer space, $n=1,2, \ldots, m=0,1, \ldots[16]$. E.g. for $n=2, m=\infty, \rho=a c /(2 f)<1$, we obtain:

$$
\begin{gathered}
D(x)=1+\frac{2 \rho e^{-(1-\rho) f x}}{(1-\rho)(1-2 \rho)}-\frac{2 \rho^{2} p_{0}}{\sqrt{\rho(4+\rho)}} \times \\
{\left[\frac{1-b_{1}}{\left(1-b_{1}\right)\left(1-b_{1}-\rho\right)} e^{-b_{1} f x}-\frac{1-b_{2}}{\left(1-b_{2}\right)\left(1-b_{2}-\rho\right)} e^{-b_{2} f x}\right],}
\end{gathered}
$$

if $\rho \neq 1 / 2$, and

$$
D(x)=1-\frac{2}{27}\left[(3 f x+10) e^{-f x / 2}-e^{-2 f x}\right], \text { if } \rho=1 / 2,
$$

where $p_{0}=(1-\rho) /(1+\rho)$ and $b_{1}, b_{2}$ are calculated by relations (9).

\section{Loss Characteristics of Systems with Random Volume Customers}

In classical queueing theory, a unique loss characteristic is $P_{\text {loss }}$, i.e. the relative part of number of customers that were lost in steady state during infinite time interval. In terms of our theory, each customer in a classical system has the volume equals 1 .

Let $D_{V}(x)$ be steady-state DF of the total customers' volume in a queueing system with limited (by $V$ ) buffer space $\left(D_{V}(x)=1\right.$, if $x>V$ ).

Generally, $P_{\text {loss }}$ can be obtained from the stability conditions, i.e. from the fact that (in steady state) the mean number of customers accepted to the system during some time interval must be equal to the mean number of customers completing their service during the same interval. E.g. by this way, relation (3) were obtained. For the system with Poisson arrival process, in which only buffer space is limited and there are no other limitations, we can determine $P_{\text {loss }}$ by the following relation [13], [17]:

$$
P_{\text {loss }}=1-\int_{0}^{V} D_{V}(V-x) d L(x),
$$

where $L(x)$ is DF of RV $\zeta$. It is clear that $P_{\text {loss }}$ is also a performance characteristic of systems with random volume customers. But it cannot take into account the fact of difference between loss probabilities for customers of different volumes.

Other (may be more objective) loss characteristic is probability $Q_{\text {loss }}$ of volume unit losing [13], [17], i.e. the relative part of total volume of customers that were lost during infinite time interval. For the system with Poisson arrival process and without other limitations except of buffer space one, this probability can be calculated by the following relation:

$$
Q_{\text {loss }}=1-\frac{1}{\varphi_{1}} \int_{0}^{V} x D_{V}(V-x) d L(x),
$$

where $\varphi_{1}=\mathbf{E} \zeta=\int_{0}^{\infty} x d L(x)$ is the mean customer's volume. E.g., for system $M / M / 1 /(\infty, V)$ with independent customer's volume and service time, we obtain from relation (4): 


$$
Q_{\mathrm{loss}}=p_{0} \int_{0}^{V} x\left[A_{n}(V-x)+\frac{n^{n}}{n !} B_{n}(V-x)\right] d L(x) .
$$

It is clear that, for fixed $n$, we can easily calculate $Q_{\text {loss }}$ in the case of exponentially distributed service time (see Section 3).

Generally, $Q_{\text {loss }}$ can be also obtained from the stability conditions, i.e. from the fact that (in steady state) the mean total volume of customers accepted to the system during some time interval must be equal to the mean total volume of customers completing their service during the same interval. However, in this case, appropriate calculations are significantly more complicated than in the case of $P_{\text {loss }}$ ones.

Denote by $\mathbf{E}(\zeta \mid A)$ a conditional mean of RV $\zeta$ under condition that an event $A$ $(\mathbf{P}\{A\}>0)$ takes place (see e.g. [18, p. 70]).

Using theory of regenerative processes [5], [19], we can prove the following statement.

Theorem. Assume that, for a system under consideration, stochastic processes $\sigma(t)$ and $\eta(t)$ are regenerative with finite mean value of regeneration cycle. Let $A$ be a random event that means a loss of arriving customer. Then, characteristics $Q_{\text {loss }}$ and $P_{\text {loss }}$ are connected by the relation $Q_{\text {loss }}=\frac{\mathbf{E}(\zeta \mid A)}{\varphi_{1}} P_{\text {loss }}$, where $\mathbf{E}(\zeta \mid A)$ is a conditional mean customer's volume under condition $A$ (i.e. $\mathbf{E}(\zeta \mid A)$ is a mean volume of losing customers).

Proof. Let $n_{j}$ be the number of arriving customers during $j$ th regeneration cycle, $n_{j}^{*}$ be the number of losing customers during this cycle, $j=1,2, \ldots, N, v_{i j}^{*}$ be the volume of $i$ th losing customer within this cycle, $i=1,2, \ldots, n_{j}^{*}, V_{j}^{*}$ be the total losing volume within this cycle: $V_{j}^{*}=\sum_{i=1}^{n_{j}^{*}} v_{i j}^{*}$.

As it follows from the theory of regenerative processes [19], we have $N^{-1} \sum_{j=1}^{N} V_{j}^{*} \stackrel{\text { a.s. }}{\longrightarrow} \mathbf{E} \chi^{*}$ when $N \rightarrow \infty$, where $\mathrm{RV} \chi^{*}$ is the total volume of losing customers within arbitrary regeneration cycle, $N^{-1} \sum_{j=1}^{N} n_{j}^{*} \stackrel{a . s .}{\longrightarrow} \mathbf{E} v^{*}$, where RV $v^{*}$ means the number of losing customers during a regeneration cycle. It is clear that $\chi^{*}=\mathbf{E} v^{*} \mathbf{E}(\zeta \mid A)$. By similar way, we obtain that $N^{-1} \sum_{j=1}^{N} n_{j} \stackrel{\text { a.s. }}{\longrightarrow} \mathbf{E} v$, where RV $v$ means the number of arriving customers on arbitrary regeneration cycle. Then, $\mathbf{E} \zeta \mathbf{E} v$ is the mean total volume of arriving customers on arbitrary cycle and we obtain:

$$
\frac{N^{-1} \sum_{j=1}^{N} V_{j}^{*}}{\mathbf{E} \zeta \mathbf{E} v} \stackrel{\text { a.s. }}{\longrightarrow} Q_{\text {loss }}=\frac{\mathbf{E}(\zeta \mid A)}{\mathbf{E} \zeta} \cdot \frac{\mathbf{E} v^{*}}{\mathbf{E} v}=\frac{\mathbf{E}(\zeta \mid A)}{\mathbf{E} \zeta} P_{\text {loss }}
$$

Hence, $Q_{\text {loss }} \geq P_{\text {loss }}$, if $\mathbf{E}(\zeta \mid A) \geq \mathbf{E} \zeta=\varphi_{1}$, i.e. the mean volume of losing customers is equal or greater than the mean volume of arbitrary ones. It is clear that $Q_{\text {loss }}=P_{\text {loss }}$, if the loss of a customer does not depend on his volume.

It was proved [20] that the relation $P_{\text {loss }}=1-\left(1-p_{0}\right) / \rho$ holds for one-server systems when service time does not depend on customer's volume, and the relation $Q_{\text {loss }}=1-\left(1-p_{0}\right) / \rho$ holds when service time is proportional to customer's volume. 
We can obtain loss characteristics in explicit form for some queueing systems with random volume customers. E.g. from relation (11), we can calculate probability $P_{\text {loss }}$ (see relation (6)) for system $M / M / 1 /(\infty, V)$ with independent $\mathrm{RVs} \zeta$ and $\xi$, when $\mathrm{RV} \zeta$ is distributed exponentially. The same formula takes place for processor sharing system with exponential customer's length ${ }^{1}$ distribution (with parameter $\mu$ ) and independent exponentially distributed customer's volume (with parameter $f$ ). For both these systems, we can obtain the following relation for $Q_{\text {loss }}$ (using relation (12)) [17]:

$$
Q_{\text {loss }}=\frac{p_{0} e^{-f V}}{\rho}\left[(1+\rho) e^{\rho f V}-1\right],
$$

where $p_{0}$ is determined by relation (5), $\rho=a / \mu$.

If, in processor sharing system, customer's volume has an exponential distribution with parameter $f$ and customer's length is proportional to his volume $(\xi=c \zeta, c>0)$, we obtain [17]:

$$
\begin{gathered}
P_{\text {loss }}=p_{0} e^{-f V} \cosh (\sqrt{\rho} f V), \\
Q_{\text {loss }}=\frac{p_{0} e^{-f V}}{\sqrt{\rho}}[\sinh (\sqrt{\rho} f V)+\sqrt{\rho} \cosh (\sqrt{\rho} f V)],
\end{gathered}
$$

where $\rho=a c / f$ and

$$
p_{0}= \begin{cases}\frac{1-\rho}{1-\sqrt{\rho} e^{-f V}[\sinh (\sqrt{\rho} f V)+\sqrt{\rho} \cosh (\sqrt{\rho} f V)]}, & \text { if } \rho \neq 1, \\ \frac{4}{3+2 f V+e^{-2 f V}}, & \text { if } \rho=1 .\end{cases}
$$

For systems with $V<\infty$ and dependent $\operatorname{RVs} \zeta$ and $\xi$, we, as a rule, cannot exactly determine loss characteristics. An exception are some models without waiting places, e.g. $M / G / n /(0, V)$ or processor sharing systems [22], [23]. But, in this case, we often can estimate these characteristics with the help of models with unlimited buffer space.

Let QS be the system with Poisson arrival process and without any limitations (there are no losses in it). Let $\mathrm{QS}_{V}$ be the system, which differs from QS in limitation (by $V, V<\infty$ ) of buffer space capacity, only. Assume that steady state exists for both systems. Denote by $D(x)$ and $D_{V}(x)$ DFs of the steady-state total volume in systems $\mathrm{QS}$ and $\mathrm{QS}_{V}$, respectively.

It can be proved (see e.g. [17]) that inequality $D(x) \leq D_{V}(x)$ holds for all real $x$. Then, we obtain the following inequalities:

$$
\begin{gathered}
P_{\text {loss }}=1-\int_{0}^{V} D_{V}(V-x) d L(x) \leq 1-\int_{0}^{V} D(V-x) d L(x)=P_{\text {loss }}^{*} \\
Q_{\text {loss }}=1-\frac{1}{\varphi_{1}} \int_{0}^{V} x D_{V}(V-x) d L(x) \leq 1-\frac{1}{\varphi_{1}} \int_{0}^{V} x D(V-x) d L(x)=Q_{\text {loss }}^{*} .
\end{gathered}
$$

1 In a processor sharing system, service time of the customer, under condition that there are no other customers in the system during his service, is called customer's length (see e.g. [20]). 
As it follows from these inequalities, the values $P_{\text {loss }}^{*}$ and $Q_{\text {loss }}^{*}$ can be interpreted as upper boundaries for $P_{\text {loss }}$ and $Q_{\text {loss }}$, respectively, when DF $D(x)$ is known.

Unfortunately, calculation of these characteristics is often very complicated, because we have function $D(x)$ very rarely in explicit form. For this function approximation, we often can use incomplete $\operatorname{Gamma}$ function $\gamma(p, b x)$ and $\operatorname{Gamma}$ function $\Gamma(p)=\gamma(p, \infty)$. E.g. in both inequalities, we sometimes can approximate $D(x)$ by the function [13]:

$$
D^{*}(x)=p_{0}+\left(1-p_{0}\right) \frac{\gamma(p, b x)}{\Gamma(p)}
$$

The values of parameters $p$ and $b$ we choose so that the first and second moments of the approximate distribution are equal to the first $\left(\delta_{1}\right)$ and second $\left(\delta_{2}\right)$ moments of DF $D(x)$. Then, we have:

$$
p=\frac{\delta_{1}^{2}}{\left(1-p_{0}\right) \delta_{2}-\delta_{1}^{2}}, b=\frac{\left(1-p_{0}\right) \delta_{1}}{\left(1-p_{0}\right) \delta_{2}-\delta_{1}^{2}} .
$$

E.g., if $\mathrm{RV} \zeta$ is absolute continuous, we obtain for $Q_{\text {loss }}^{*}$ the following approximate relation [24]:

$$
Q_{\mathrm{loss}}^{*} \approx 1-\frac{1}{\varphi_{1}} \int_{0}^{V} x\left[p_{0}+\left(1-p_{0}\right) \frac{\gamma(p, b(V-x))}{\Gamma(p)}\right] l(x) d x,
$$

where $l(x)$ is density of RV $\zeta$. To calculate $P_{\text {loss }}^{*}$ and $Q_{\text {loss }}^{*}$, we can use methods of numerical integration (see e.g. [25]).

\section{Analog of Little's Formula}

Little's formula $\mathbf{E} \eta=\lambda \mathbf{E} v$, where $\eta$ is number of customers present in the system in steady state, $\lambda$ is intensity of (stationary) arrival process, $v$ is sojourn time of a customer in the system, is one of the most significant results of classical queueing theory. It holds for arbitrary steady-state system without losing of customers.

Consider an arbitrary queueing system with random volume customers. Assume that RV $\zeta$ means customer's volume and $\xi$ his service time. Denote by $\alpha_{11}=\mathbf{E}(\zeta \xi)$ the mixed moment of $(1+1)$ th order of RVs $\zeta$ and $\xi$. Assume that arrival process is stationary with intensity $\lambda$. Let $w$ be queueing time of a customer, hence, $v=w+\xi$, where $\operatorname{RVs} w$ and $\xi$ are independent. Let $\sigma$ be the total volume of customers present in the system in steady state. It was proved (see [26], [27]) that the following relation named an analog of Little's formula holds:

$$
\mathbf{E} \sigma=\lambda(\mathbf{E} w \cdot \mathbf{E} \zeta+\mathbf{E}(\zeta \xi)) .
$$

Consider, as an example, two steady-state systems of $M / M / 1 / \infty$ type: $\mathrm{QS}_{1}$ and $\mathrm{QS}_{2}$. Let $a$ be parameter (intensity) of arrival process in both systems. Assume that customer's volume is distributed exponentially with parameter $f$ in each of them, i.e. $L(x)=\mathbf{P}\{\zeta<x\}=1-e^{-f x}$. Assume that customer's volume and service time are independent in $\mathrm{QS}_{1}$. Denote by $\mu$ parameter of service time in it, i.e. $B_{1}(t)=1-$ 
$e^{-\mu t}$. Assume that, in $\mathrm{QS}_{2}$, service time is proportional to customer's volume: $\xi=c \zeta, c>0$. Then, we have for this system:

$$
B_{2}(t)=\mathbf{P}\{\xi<t\}=\mathbf{P}\{c \zeta<t\}=\mathbf{P}\{\zeta<t / c\}=1-e^{-f t / c} .
$$

Assume that equality $\mu=f / c$ holds. Then, we obtain $B_{1}(t)=B_{2}(t)$. It means that the systems $\mathrm{QS}_{1}$ and $\mathrm{QS}_{2}$ are identical from classical queueing theory point of view, i.e. distributions of all RVs characterizing these systems (e.g. queueing time, sojourn time, number of customers present in the system) are the same in both systems. But this assertion becomes wrong for mean values $\mathbf{E} \sigma^{(1)}$ and $\mathbf{E} \sigma^{(2)}$ of total volumes for systems $\mathrm{QS}_{1}$ and $\mathrm{QS}_{2}$, respectively.

Indeed, $\mathrm{RVs} \zeta$ and $\xi$ are independent in $\mathrm{QS}_{1}$. Then, for their mixed moment $\alpha_{11}^{(1)}$, we obtain:

$$
\alpha_{11}^{(1)}=\mathbf{E}(\zeta \xi)=\mathbf{E} \zeta \cdot \mathbf{E} \xi=(f \mu)^{-1} .
$$

Classical characteristic $\mathbf{E} w$ is the same for both systems. It is known [3], [5] that $\mathbf{E} w=\rho /(\mu(1-\rho))$, where $\rho=a / \mu=a c / f$. Then, for system $\mathrm{QS}_{1}$, we have from relation (16):

$$
\mathbf{E} \sigma^{(1)}=a\left[\frac{\rho}{\mu(1-\rho)} \cdot \frac{1}{f}+\frac{1}{f} \cdot \frac{1}{\mu}\right]=\frac{1}{f} \cdot \frac{\rho}{1-\rho} .
$$

For mixed moment $\alpha_{11}^{(2)}$ of the system $\mathrm{QS}_{2}$, we have:

$$
\alpha_{11}^{(2)}=\mathbf{E}(\zeta \xi)=\mathbf{E}(\zeta \cdot c \zeta)=c \mathbf{E} \zeta^{2} .
$$

For the second moment $\mathbf{E} \zeta^{2}$ of customers' volume, we easily obtain $\mathbf{E} \zeta^{2}=2 / f^{2}$ and finally have:

$$
\mathbf{E} \sigma^{(2)}=a\left[\frac{\rho}{\mu(1-\rho)} \cdot \frac{1}{f}+\frac{2 c}{f^{2}}\right]=\frac{1}{f} \cdot \frac{\rho(2-\rho)}{1-\rho} .
$$

Therefore, mean total volume is $2-\rho$ times greater in the system $\mathrm{QS}_{2}$. It is intuitively clear, because customers of a greater volume are served for a longer time in this system.

This simple example shows that we have to take into account the dependence between RVs $\zeta$ and $\xi$, if we want correctly determine buffer space capacity while communication center (router) designing of a computer or telecommunication network.

\section{Examples of Calculations of Loss Characteristics}

Now, we shall present some exemplary calculations of loss characteristics for the chosen queueing systems with random volume customers.

\subsection{Processor sharing system $M / M / 1 /(\infty, V)$-EPS}

Consider processor sharing system in which customer's volume is distributed exponentially with parameter $\mathrm{f}$. We shall analyze two versions of this system. In first 
of them $\left(\mathrm{QS}_{1}\right)$, customer's length is independent on his volume and is exponentially distributed with parameter $\mu$. In second one $\left(\mathrm{QS}_{2}\right)$, customer's length is proportional to his volume $(\xi=c \zeta, c>0)$ i.e. it is distributed exponentially with parameter $f / c$. If we assume that $\mu=f / c$, we obtain that the systems $\mathrm{QS}_{1}$ and $\mathrm{QS}_{2}$ become identical from the classical queueing theory point of view. But, of course, loss characteristics are not the same for these systems, because the dependence between RVs $\zeta$ and $\xi$ has a substantial influence on their values. Indeed, in Sections 3 and 4, we obtained explicit relations for loss characteristics of the systems $\mathrm{QS}_{1}$ (relations (6) and (13)) and $\mathrm{QS}_{2}$ (relations (14) and (15)). Now, we present some numerical computations under assumption that $a=1, f=2$ for both systems, $\mu=2$ for $\mathrm{QS}_{1}$ and $c=1$ for $\mathrm{QS}_{2}$, i.e. $\rho=0.5$ for both systems. The results of computations are presented in Fig. 3 (for $\mathrm{QS}_{1}$ ) and Fig. 4 (for $\mathrm{QS}_{2}$ ).

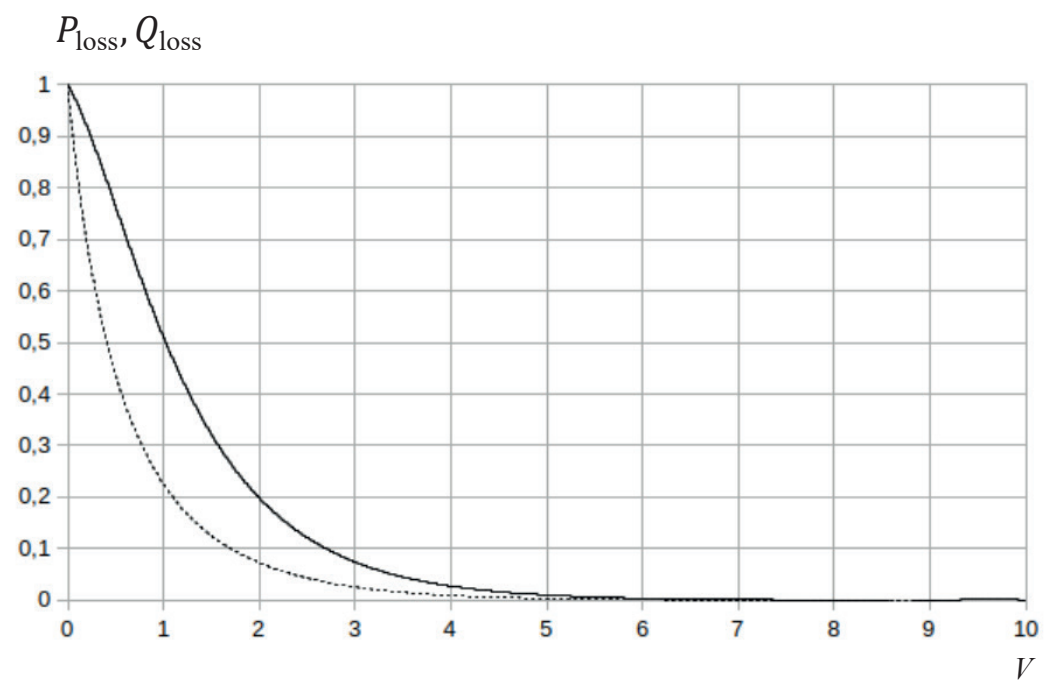

Fig. 3. Loss characteristics for system $M / M / 1 /(\infty, V)-E P S$ with independent customer's

\subsection{System $M / M / 1 /(\infty, V)$}

Consider single-server queueing system in which customer's volume is distributed exponentially with parameter $f$. We shall also analyze two versions of this system $\left(\mathrm{QS}_{1}\right.$ and $\left.\mathrm{QS}_{2}\right)$. In the first of them, customer's service time is independent of his volume and distributed exponentially with parameter $\mu$. In the second one, service time is proportional to customer's volume $(\xi=c \zeta)$. If we assume that $\mu=f / c$, these two models are also equivalent from the classical point of view. 


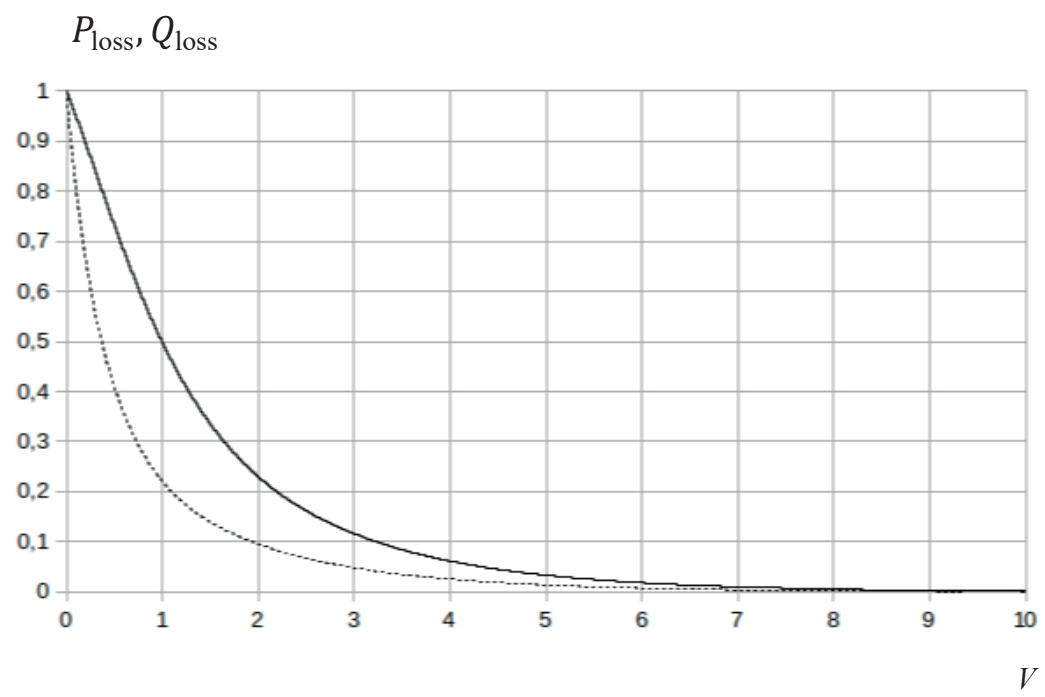

Fig. 4. Loss characteristics for system $M / M / 1 /(\infty, V)-E P S$ with customer's length proportional to his volume

It is clear that we can't calculate exact loss characteristics for the second version of the system, because its DF $D_{V}(x)$ is unknown in explicit form. But, for both versions, we can calculate approximations of $D_{V}(x)$ (see Section 4). These approximations are based on the use of incomplete gamma function with parameters that depend on the values of the first $\left(\delta_{1}\right)$ and second $\left(\delta_{2}\right)$ moments of analogous system $M / M / 1 / \infty$ with unlimited buffer space, i.e. $V=\infty$ (see Section 3 ), under assumption $\rho=a / \mu=\mathrm{ac} / f<1$. For system $\mathrm{QS}_{1}$ from relation (10), we easily obtain:

$$
\delta_{1}=\frac{\rho}{f} \cdot \frac{1}{1-\rho}, \delta_{2}=\frac{2 \rho}{f^{2}} \cdot \frac{1}{(1-\rho)^{2}} .
$$

For system $\mathrm{QS}_{2}$, we have analogously from relation (8):

$$
\delta_{1}=\frac{\rho}{f} \cdot \frac{2-\rho}{1-\rho}, \delta_{2}=\frac{2 \rho}{f^{2}} \cdot \frac{3+\rho^{3}-\rho^{2}-2 \rho}{(1-\rho)^{2}} .
$$

Now, we present some numerical computations. Assume that $a=1, f=2$. Then, $\rho=0.5$. The results of calculation for system $\mathrm{QS}_{1}$ are presented in Fig. 5. Proper results for $\mathrm{QS}_{2}$ are shown in Fig. 6.

Obtained numerical results show that a character of dependency between customer's service time and his volume has substantial influence on estimators of loss characteristics, and we have to take it into account during the process of computer systems designing. E.g. if we want to design computer system having the same input characteristics and want to have maximal volume unit loss probability less than $10^{-4}$, then, in the case of $\mathrm{QS}_{1}$, we have to set value $V$ at least equal to 10 , and, in the case of $\mathrm{QS}_{2}$, to set it equal at least to 12 . Of course, we can see that $Q_{\text {loss }}$ characteristics are 
greater than $P_{\text {loss. }}$. Numerical computations of loss characteristics and other performance ones for various types of queueing systems with random volume customers (also with non-identical servers) can be found in [23], [28] and [29].

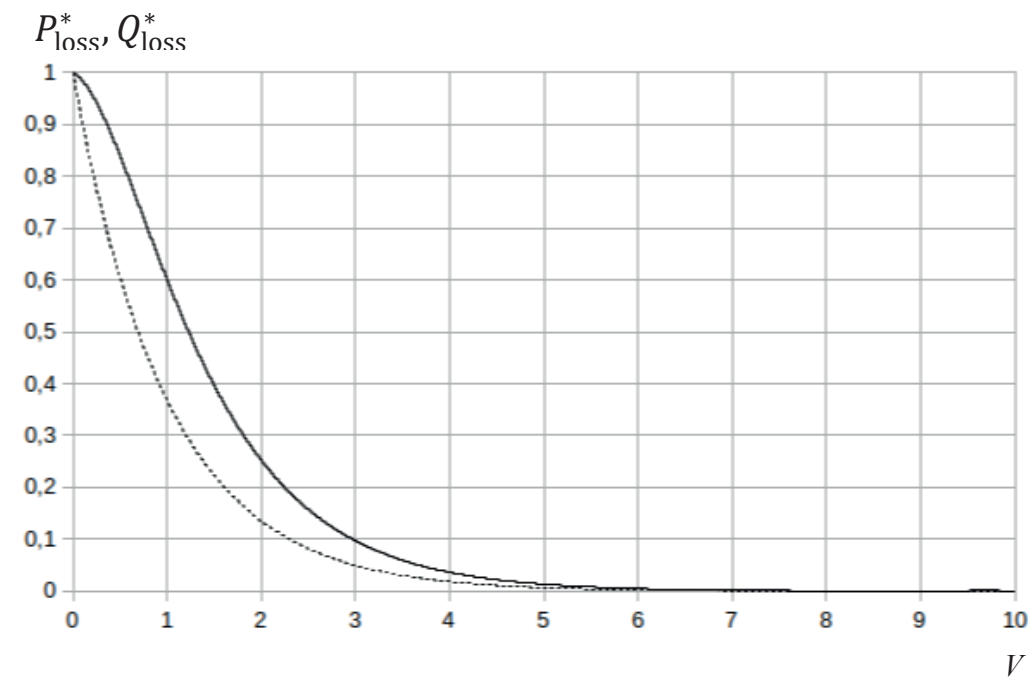

Fig. 5. Loss characteristics for system $M / M / 1 /(\infty, V)$ with independent customer's volume and his service time (approximation)

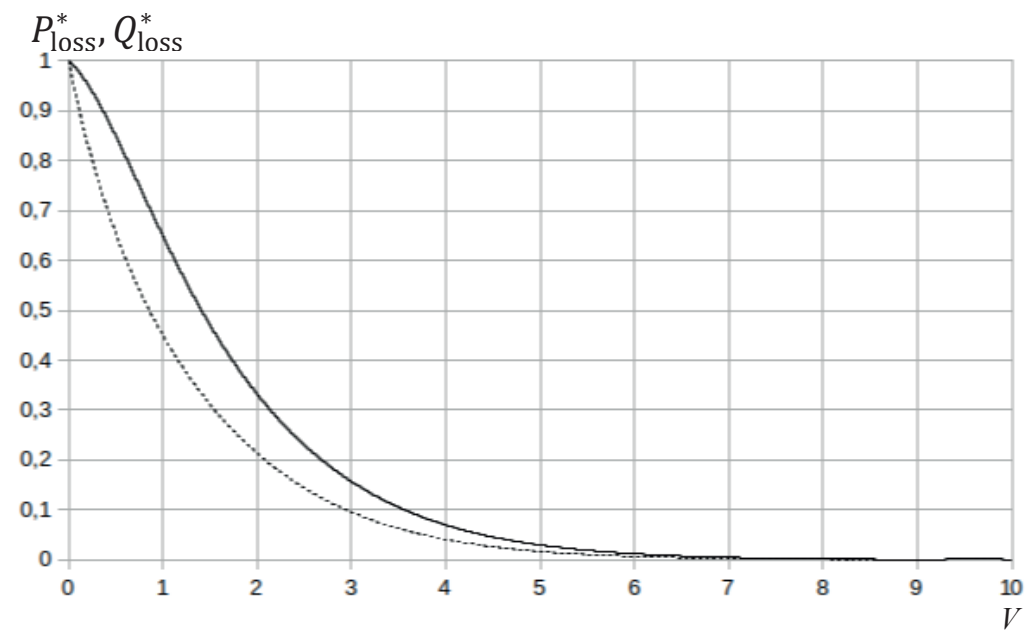

Fig. 6. Loss characteristics for system $M / M / 1 /(\infty, V)$ with service time proportional to customer's volume (approximation)

\section{Conclusion}

In the paper, we focus on models of queueing systems with random volume customers. We show that analysis of these models needs some extensions of mathematical tools known from the classical queueing theory. 
We also discuss the most important problems that are connected with determining of distribution functions of the customers' total volume in such models and obtaining of their loss characteristics. We additionally present results of investigations for some models of such systems. We point out that the character of dependency between customer's volume and his service time has incontestable influence on performance characteristics of analyzed models that was shown in some numerical examples. Moreover, we present some practical approximation methods of calculating estimators of loss characteristics in the case when it is not possible to obtain explicit formulae.

These methods can be used in computer systems designing, when we determine needed buffer space capacity of the system.

\section{References}

[1] B. V. Gnedenko, I. N. Kovalenko, Introduction to Queueing Theory, Birkhauser, Cambridge, 1989.

[2] L. Kleinrock, Queueing Systems, Volume 1: Theory, Wiley, New York, 1976.

[3] P. P. Bocharov, C. D’Apice, A. V. Pechinkin, S. Salerno, Queueing Theory, Utrecht-Boston: VSP, 2004.

[4] L. Breuer, D. Baum, An Introduction to Queueing Theory and Matrix-Analytic Methods, Springer, Dordrecht, 2005.

[5] L. Lakatos, L. Szeidl, M. Telek, Introduction to Queueing Systems with Telecommunication Applications, Springer, New York, 2013.

[6] V. N. Roginsky, A. D. Kharkevich, M. A. Shneps, Communication Networks Theory, Radio i Svyaz', Moscow, 1981 (in Russian).

[7] M. Schwartz, Computer-Communication Network Design and Analysis, Prentice-Hall, New Jersey, 1977.

[8] M. Schwartz, Telecommunication Networks: Protocols, Modeling and Analysis, Addison-Wesley Publishing Company, 1987.

[9] N. B. Zeliger, O. S. Chougreev, G. G. Yanovsky, Design of Networks and Systems of Discrete Messages Transmission, Radio i Svyaz', Moscow, 1984 (in Russian)

[10] A. M. Alexandrov, B. A. Kaz, Non-homogeneous demands flow service, Izvestiya AN SSSR. Technicheskaya Kibernetika, no. 2, 1973, pp. 47-53 (in Russian).

[11] B. Sengupta, The spatial requirements of an $M / G / 1$ queue, or: how to design for buffer space, In: F. Baccelli and G. Fayolle (eds.) Modeling and Performance Evaluation Methodology, LNCS, vol. 60, Springer, Heidelberg, 1984, pp. 547-562. 
[12] O. M. Tikhonenko, Queueing Models in Computer Systems, Universitetskoe, Minsk, 1990 (in Russian).

[13] O. Tikhonenko, Computer Systems Probability Analysis, Akademicka Oficyna Wydawnicza EXIT, Warsaw, 2006 (in Polish).

[14] O. Tikhonenko, M. Kawecka, Queueing Systems with Customers of Random Space Requirements, Studia Informatica, vol. 33, no. 3A (107), Gliwice 2012, pp. 19-28 (in Polish).

[15] O. Tikhonenko, W. M. Kempa, Performance Evaluation of an $M / G / n$-type Queue with Bounded Capacity and Packet Dropping, International Journal of Applied Mathematics and Computer Science, Vol. 26, no. 4, 2016, pp. 841-854.

[16] O. Tikhonenko, M. Kawecka, Total Volume Distribution for Multiserver Queueing systems with Random Capacity Demands, Communications in Computer and Information Science, vol. 370, Springer, 2013, pp. 394-405.

[17] O. Tikhonenko, Determination of Loss Characteristics in Queueing Systems with Demands of Random Space Requirement, Communications in Computer and Information Science, vol. 564, Springer Internetional Publishing Switzerland, 2015, pp. 209-215.

[18] A. A. Borovkov, Probability Theory, Springer-Verlag, London, 2013.

[19] S. Asmussen, Applied Probability and Queues, 2d ed., Springer-Verlag, New York, 2003.

[20] E. Morozov, R. Nekrasova, L. Potakhina, O. Tikhonenko, Asymptotic analysis of queueing systems with finite buffer space, Communications in Computer and Information Science, vol. 431, Springer International Publishing Switzerland, 2014, pp. 223-232.

[21] S. F. Yashkov, A. S. Yashkova, Processor Sharing: a Survey of the Mathematical Theory, Automation and Remote Control, vol. 68, no. 9, 2007, pp. 1662-1731.

[22] O. M. Tikhonenko, Generalized Erlang Problem for Service systems with Finite total Capacity, Problems of Information Transmission, vol. 41, no. 3, 2005, pp. 243-253.

[23] O. M. Tikhonenko, Queueing systems with processor sharing and limited resources, Automation and Remote Control, vol. 71, no. 5, 2010, pp. 803815.

[24] O. Tikhonenko, M. Ziółkowski, Single-Server Queueing System with External and Internal Customers, Bulletin of the Polish Academy of Sciences, Technical Sciences, vol. 66, no. 4, 2018, pp. 539-551.

[25] Y. L. Luke, Mathematical Functions and their Approximations, Academic Press Inc., New York, 1975. 
[26] O. M. Tikhonenko, An analog of Little's formula for queuing systems with non-uniform customers, Automation and Remote Control, vol. 57, no. 1, 1996, pp. 85-88.

[27] E. Morozov, L. Potakhina, O. Tikhonenko, Regenerative analysis of a system with a random volume of customers, Communications in Computer and Information Science, vol. 638, Springer International Publishing Switzerland, 2016, pp. 261-272.

[28] O. Tikhonenko, M. Ziółkowski, M. Kurkowski, $M / \vec{G} / n /(0, V)$ Erlang Queueing System with Non-Homogeneous Customers, Non-Identical Servers and Limited Memory Space, Bulletin of the Polish Academy of Sciences, Technical Sciences, vol. 67, no. 3, 2019, pp. 489-500.

[29] M. Ziółkowski, $M / \vec{G} / n / 0$ Erlang Queueing System with Heterogeneous Servers and Non-Homogeneous Customers, Bulletin of the Polish Academy of Sciences, Technical Sciences, vol. 66, no. 1, 2018, pp. 59-66. 